\title{
Types of left brachiocephalic vein aberrations detected during cardiac implantable electronic device implantation procedures
}

\author{
R. Steckiewicz'1 ${ }^{1}$, P. Stolarz², E.B. Świętoń ${ }^{1}$ \\ ${ }^{1} 1^{\text {st }}$ Department of Cardiology, Central University Hospital in Warsaw, Poland \\ $21^{\text {st }}$ Chair and Department of Cardiology, Medical University of Warsaw, Poland \\ [Received: 10 January 2020; Accepted: 28 May 2020]
}

Background: Cardiac implantable electronic device (CIED) implantation procedures with transvenous lead placement afford an opportunity to observe vascular anatomic variations. The course of CIED implantation depends largely on morphometric and topographic characteristics of the relevant brachiocephalic vein (BCV), which is the left $B C V$ in the case of lead insertion via the left clavipectoral triangle. This study aims to present left BCV anomalies arising from abnormal systemic vein embryogenesis and encountered during CIED implantation.

Materials and methods: Venograms obtained during CIED implantation procedures and illustrating left $B C V$ topography/morphometry were analysed retrospectively for two types of anomalies: anomalies of the left BCV itself (data from the period 2014-2018) and a combination of left BCV variations with a persistent left superior vena cava (PLSVC); since the latter instances are rare, the analysed period was longer (2003-2018).

Results: Analysis of data from the first, 5-year-long, period included data from a group of 1812 patients and revealed $5(0.3 \%)$ cases of developmental left-BCV anomalies (3 double left $B C V$ and 2 cases of a single subaortic left $B C V$ ). The 16-year-long analysed period included 6110 CIED implantation procedures, which showed 12 (0.2\%) cases of PLSVC including 4 (33\%) cases of left BCV agenesis. Conclusions: The analysed venograms rarely showed isolated left- $B C V$ aberrations $(0.3 \%)$, with the combination of left-BCV agenesis and PLSVC being much more common (33\%). The morphometry and/or topography of aberrant left-BCV may result in difficulties during cardiac lead insertion. (Folia Morphol 2021; 80, 2: 317-323)

Key words: aberrant left brachiocephalic vein, persistent left superior vena cava, venography, lead implantation, cardiac implantable electronic device (CIED)

\section{INTRODUCTION}

Procedures utilising transvenous catheter insertion, including cardiac implantable electronic device (CIED) implantation, afford an opportunity to detect vascular anatomy variations, such as the natural physiological individual variations in the shape or course of vessels and vascular anomalies due to disturbances during embryogenesis $[1,5,11,17]$. The normal

Address for correspondence: R. Steckiewicz, MD, PhD, $1^{\text {st }}$ Department of Cardiology, Central University Hospital in Warsaw, ul. Banacha $1 \mathrm{~A}$, 02-097 Warszawa, Poland, e-mail: r.steckiewicz@pro.onet.pl

This article is available in open access under Creative Common Attribution-Non-Commercial-No Derivatives 4.0 International (CC BY-NC-ND 4.0) license, allowing to download articles and share them with others as long as they credit the authors and the publisher, but without permission to change them in any way or use them commercially. 
course of said procedures depends on favourable morphometry and topography of the brachiocephalic vein $(B C V)$, also known as the innominate vein, which is a systemic vein.

Disturbances in systemic vein embryogenesis result in vascular anomalies. In the case of left BCV such anomalies exhibit considerable anatomical variations in comparison with a normal left BCV. These anomalies may manifest as variations in the course of the vein through the anterior mediastinum and its spatial relationship to adjacent anatomical structures. Another result of disturbed venous embryogenesis can be the presence of an accessory vessel which is a doubled version of the left $B C V[4,15,18,29]$.

The rates of detected left-BCV anomalies depend on the evaluated population and the used imaging technique. Left-BCV anomalies are detected in approximately $1 \%$ of patients with congenital heart defects, such as tetralogy of Fallot, atrial septal defect, and ventricular septal defect. In the population without heart defects the estimated prevalence of left-BCV anomalies is below $0.4 \%$. Developmental anomalies of the left BCV may occur in isolation or coexist with various variations of the persistent left superior vena cava (PLSVC). This type of systemic vein aberrations is found in $0.3-0.5 \%$ of individuals from the general population $[3,12,14,19,26]$.

Unlike in the case of left BCVs with a typical topography, anatomical variations of this vessel (depending on their nature and extent), may facilitate inflicting inadvertent damage to the vessel itself and to the adjacent structures during certain transvascular procedures $[2,9,10,13,22]$. This is particularly likely if the vascular variation had been asymptomatic prior to the procedure and is detected only during a CIED implantation procedure, as was the case in the examples presented below.

This paper presents our records on rare, and sometimes very rare, developmental anomalies of the left BCV observed over a number of years. The enclosed images illustrate the nature of the detected anomalies (i.e. they are a radiographic representation of vascular morphometry and topography).

\section{MATERIALS AND METHODS}

This study is a retrospective assessment of the imaging records from venography procedures performed during de-novo CIED implantation procedures involving cardiac lead insertion through the systemic veins via a venous access in the left clavipectoral triangle. The indication for contrast-enhanced venography had been intra-operative problems with lead advancement, which needed to be explained and solved.

The material presented below has been classified into two types of disturbed left BCV embryogenesis: - developmental anomalies involving only the left BCV, detected over the period 2014-2018;

- developmental anomalies of the left BCV in combination with the presence of PLSVC, detected over the period of 2003-2018.

The images of left-BCV variations presented in this paper have been selected to best illustrate characteristic examples of the relevant vascular anatomical variants.

During most of the procedures, venography involved selective contrast administration, directly via cephalic vein cutdown or axillary vein/subclavian vein puncture. This helped limit the volume of the contrast agent being administered while at the same time ensuring a more thorough filling of mediastinal vein lumina.

Our statistical analysis used numerical variables in the form of mean values, standard deviations. This study had been approved by the Institutional Review Board.

\section{RESULTS}

Table 1 provides an overview of the types of left$-B C V$ anomalies presented in this paper in terms of: patients' age at the time of first CIED implantation, procedure date, electrocardiographic indications for CIED implantation and the CIED types.

Analysis of data from the first, 5-year-long (2014-2018), period included CIED implantation procedures performed in a total of 1812 patients. Among those, there were $5(0.3 \%)$ cases of developmental left-BCV anomalies.

Over the analysed 5-year period, there were 3 cases of a double left BCV, 2 of which were detected during de-novo CIED implantation procedures (including that illustrated in Fig. 1A). The third case of a double left BCV was detected during a procedure involving the addition of another cardiac lead to a CIED that had been implanted years earlier. The venography in this case additionally showed the patency of the vein in whose lumen the previous cardiac lead resided (Fig. 1B).

In the detected cases of double left BCV, the main and duplicated vessel differed in their diameters, with only the main ('upper') vessel's course and morpho- 
Table 1. Venous anomaly types

\begin{tabular}{|c|c|c|c|c|c|}
\hline Type of BCV anomaly & Sex & Age* $^{*}$ & ECG-based indications & Procedure date & CIED type \\
\hline \multirow[t]{3}{*}{ Double BCV } & Female & 78 & $\mathrm{VT}$ & 2014 & ICD VR \\
\hline & Male & 74 & $A F, A V B$ & 2015 & Wl \\
\hline & Male & 82 & SSS+PAF & 2017 & $\mathrm{DDD}$ \\
\hline \multirow[t]{2}{*}{ Subaortal BCV } & Male & 87 & AF, AVB, EF 17\% & 2017 (ineffective procedure) & Indications for ICD VR \\
\hline & Male & 81 & SF, AVB & 2018 & Wl \\
\hline \multirow[t]{4}{*}{ Left BCV + Double SVC } & Male & 41 & SSS & 2003 & AAl \\
\hline & Female & 90 & $\mathrm{AF}+\mathrm{CHB}$ & 2004 & WI \\
\hline & Male & 63 & $\mathrm{SR}+\mathrm{CHB}$ & 2015 & $\mathrm{DDD}$ \\
\hline & Male & 67 & $\mathrm{VT}$ & 2016 & ICD VR \\
\hline \multirow[t]{4}{*}{ Double SVC without BCV } & Female & 77 & TBS & 2006 & $\mathrm{DDD}$ \\
\hline & Female & 80 & $\mathrm{SR}+\mathrm{CHB}$ & 2006 & WI \\
\hline & Male & 61 & TBS & 2007 & $\mathrm{DDD}$ \\
\hline & Female & 67 & $\mathrm{AF}+\mathrm{CHB}$ & 2017 & Wl \\
\hline \multirow[t]{4}{*}{ Single persistent left SVC } & Female & 52 & $\mathrm{SR}+\mathrm{CHB}$ & 2009 & $\mathrm{DDD}$ \\
\hline & Male & 67 & $\mathrm{SR}+\mathrm{CHB}$ & 2013 & WI \\
\hline & Female & 66 & $\mathrm{VT}+\mathrm{AF}$ & 2014 & ICD VR \\
\hline & Male & 63 & $\mathrm{AF}+\mathrm{CHB}$ & 2015 & WI \\
\hline
\end{tabular}

*Patients' age at the time of first cardiac implantable electronic device (CIED) implantation; AAI — single-chamber atrial pacemaker; AF — atrial fibrillation; AVB — atrioventricular block $\left(I^{\circ} / I^{\circ}\right) ; B C V$ - brachiocephalic vein; CHB — complete heart block; DDD — dual-chamber (atrioventricular) pacemaker; EF — ejection fraction; ECG — electrocardiogram; ICD VR - single-chamber implantable cardioverter-defibrillator; PAF — paroxysmal atrial fibrillation; SVC — superior vena cava; SR — sinus rhythm; SSS — sick sinus syndrome; TBS — tachycardia -bradycardia syndrome; VT — ventricular tachycardia; VVI — single-chamber ventricular pacemaker
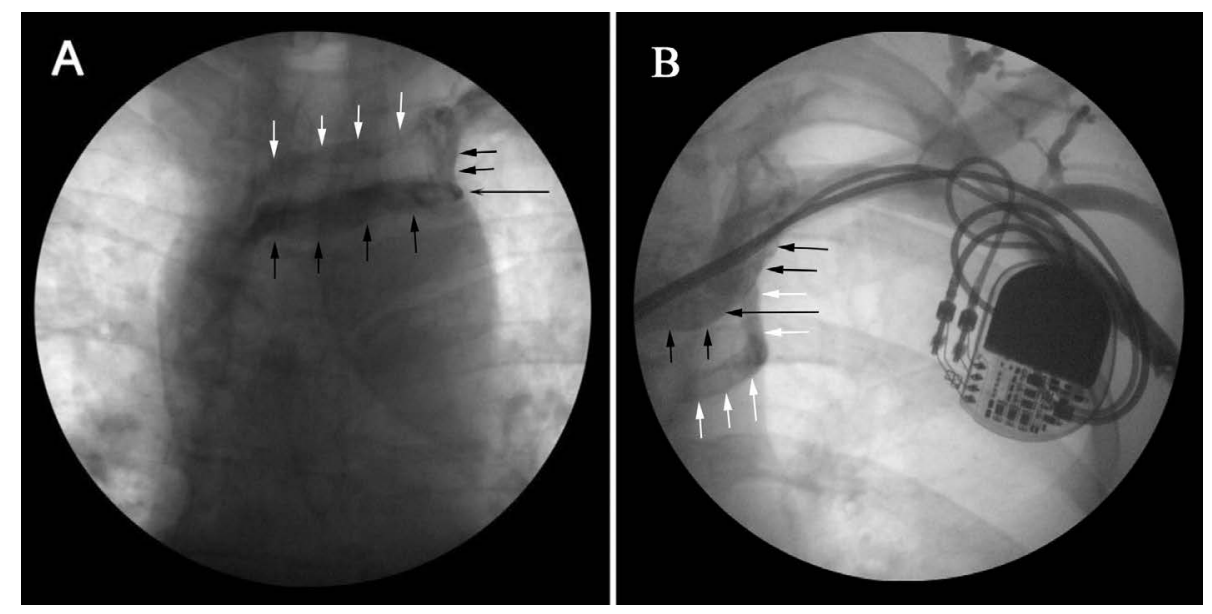

Figure 1. Differences in the lumina of the double left brachiocephalic vein variants: the main vessel (with a larger diameter and greater venous blood flow) — black arrows; the vessel with a smaller diameter and blood flow — white arrows; $\mathbf{A}$. The blood/contrast agent flowing through the lower vessel in an 82-year-old male (black arrows). The cardiac leads were later successfully threaded through the upper vessel (white arrows); B. Cardiac leads threaded through the upper vessel in a 74-year-old male (black arrows), with the lower vessel exhibiting unfavourable morphometry and topography for lead advancement (white arrows).

metry allowing cardiac leads to be threaded towards the heart.

Throughout the evaluated period, we detected 2 cases of a subaortic left BCV (Fig. 2A, B). In 1 of those patients (Fig. 2A), who had undergone aortic valve replacement in the past, the altered venous lumen morphometry precluded threading a cardiac lead beyond the site of stenosis and, thus, it was impossible to implant the CIED via a left-sided approach.

In the years 2003-2018, PLSVC was detected in 12 cases ( 6 women and 6 men), which constituted $0.2 \%$ of the $6110 \mathrm{CIED}$ implantation procedures 

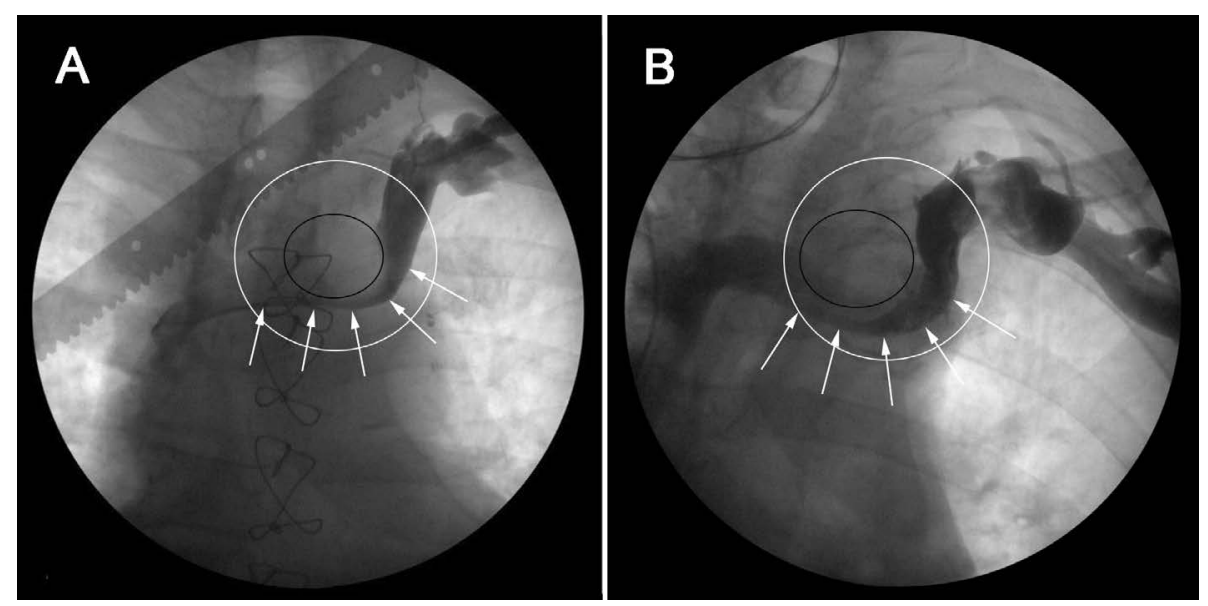

Figure 2. The general radiographic location of a subaortic left brachiocephalic vein (BCV) (white circles), including the location of the aortic arch (aortic cross-section marked with a black oval); the compressed subaortic left BCV segment (marked with arrows); A. Venous lumen compression by a dilated aorta in a 87-year-old male with a documented anatomical variation in aortic-arch arteries, involving both carotid arteries and the right subclavian artery branching off a common vessel (CT), status post aortic valve implantation, and an ascending aortic aneurysm. The oblique radiopaque stripe with a notched margin is a measuring scale; B. A radiogram from the successful implantation of a VVI pacemaker in an 81-year-old male, illustrating the venous configuration.
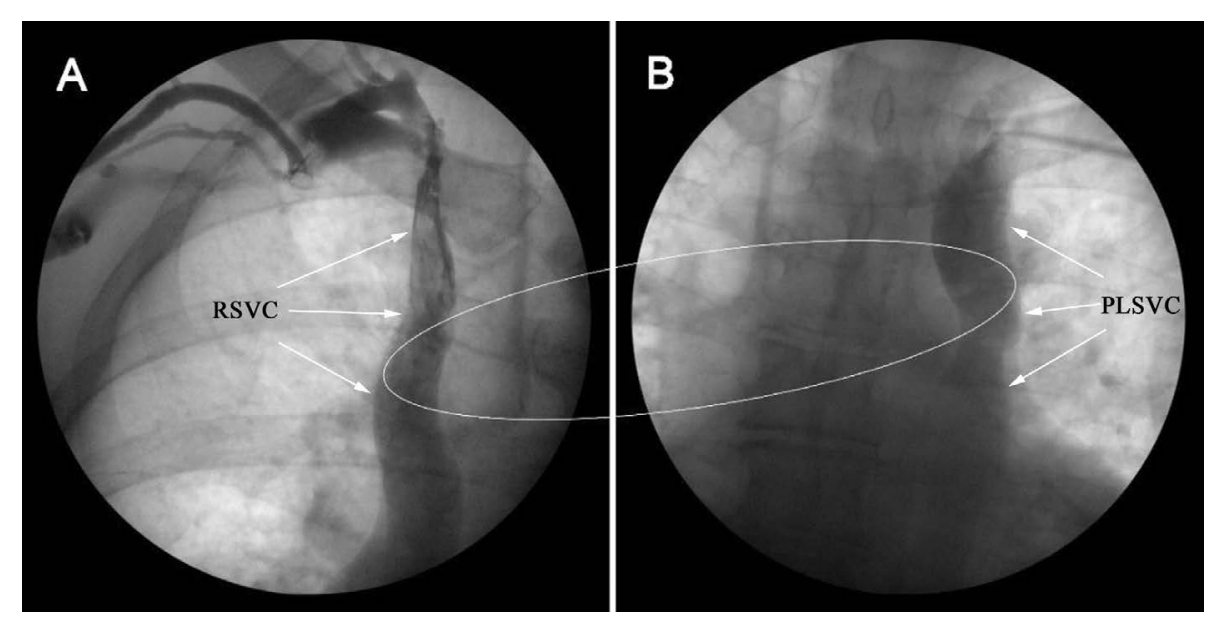

Figure 3. Persistent left superior vena cava (PLSVC) with left brachiocephalic vein (BCV) agenesis in a 61-year-old male. Intraoperative bilateral venography illustrates a lack of contrast enhancement in the left BCV potentially connecting the two superior venae cavae; A. Right superior vena cava (RSVC) morphometry illustrated with a contrast agent shows a lack of the left BCV. The site where the left BCV can be typically visualised if the vessel forms during normal embryogenesis (white oval). Marking the exact topography and morphometry of the vessel would be difficult due to inter-individual anatomical variations; B. Contrast-enhanced PLSVC also shows a lack of contrast flow through a left BCV in a situation where a left BCV bridge could be potentially present.

involving transvenous cardiac lead placement performed over this period.

In 4 PLSVC patients ( 3 women and 1 man; mean age $71 \pm 9$ years; $<0.1 \%$ ) there was concomitant left BCV agenesis (Fig. 3), with each of the two superior venae cavae having independent venous drainage.

The presence of a left BCV forming a bridge between the two superior venae cavae was detected in 4 cases (including 3 men and 1 woman; mean age $65 \pm 20$ years; one example is illustrated in Fig. 4).
In the remaining 4 cases ( 2 men and 2 women; mean age $62 \pm 7$ years), we observed a single PLSVC providing right-side thoracic drainage via an arching left BCV located in the superior mediastinum (Fig. 5).

The aberrant anatomy of systemic vessels (shown in the cases presented above and due to abnormal systemic vessel embryogenesis) proved problematic during CIED implantation procedures and lengthened procedure duration; however, there were no intraoperative complications. 

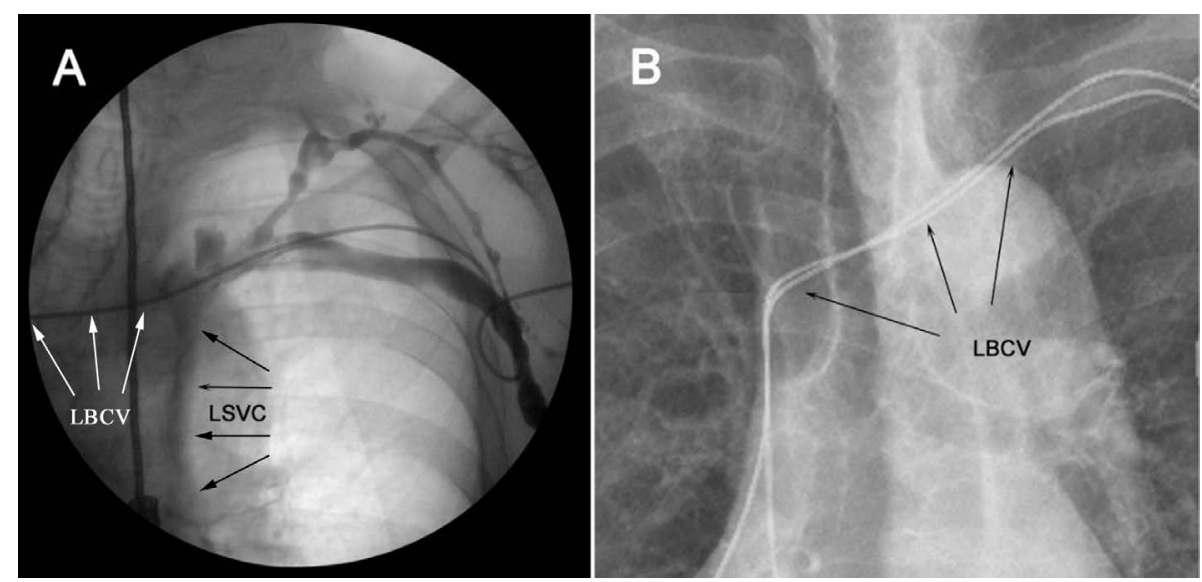

Figure 4. Persistent left superior vena cava (LSVC) with a left brachiocephalic vein (LBCV) in a 90-year-old female; $\mathbf{A}$. Venogram showing the course of the cardiac lead and demonstrating a typical course of the LBCV through the mediastinum (obliquely and inferiorly) and the presence of two superior venae cavae (fluoroscopic image obtained during intra-procedure venography); B. LBCV patency can be verified when threading a new lead is required several years after the first procedure. Post-procedure chest X-ray film illustrating the location of both cardiac leads within the LBCV.

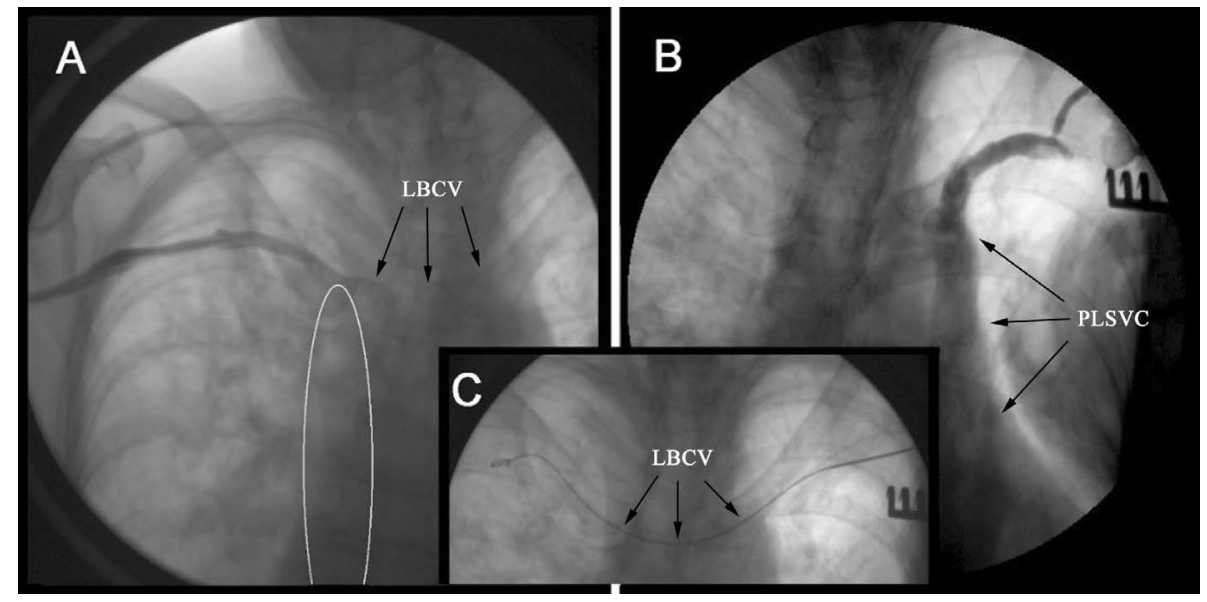

Figure 5. Single persistent left superior vena cava in a 66-year-old female; A. Right superior vena cava agenesis — documented by a lack of contrast enhancement in the vessel's potential location (white oval); B. Persistent left superior vena cava (PLSVC) morphometry poorly visualised by a retrograde flow of contrast; C. The position of a guidewire, arching between the left and the right subclavian veins, unequivocally proves the presence of a patent left brachiocephalic vein (LBCV).

\section{DISCUSSION}

In normally developing human embryos, the initially symmetrical venous system consists of paired anterior and posterior cardinal veins that drain the cephalic and caudal areas of the embryo, respectively. During further stages of physiological embryogenesis, the left and right pairs of cardinal veins anastomose inferiorly to form the left and right common cardinal veins (ducts of Cuvier). At 8 weeks of gestation, anastomoses between the left and right anterior cardinal veins lead to BCV formation. The physiological development of the systemic veins of the superior mediastinum ends with the left BCV being nearly 3 times longer than the right $B C V$. Each BCV, which is an anatomical continuation of the ipsilateral internal jugular vein and subclavian vein, drains blood from the ipsilateral side of the body.

The left-BCV is typically positioned superior to the aortic arch and anterior to the aortic arch branches (the brachiocephalic trunk, left common carotid artery, and left subclavian artery). Having crossed to the right side of the superior mediastinum, the left BCV merges with the right $\mathrm{BCV}$ to form the superior vena cava (SVC) $[21,25]$. During CIED implantation procedures, cardiac leads are typically inserted via the veins of the left clavipectoral triangle. This is also the approach preferred at our centre (utilised in approximately $97 \%$ of de novo CIED implantation procedures). 
The presence of left BCV anomalies may pose a challenge in performing transvascular procedures employing this vessel and increase the risk of their complications $[10,13,14]$. Moreover, the left BCVs that are not anomalous themselves may sometimes have tributaries, whose configurations and confluence angles differ dramatically from those typically observed. This usually poses problems during anaesthesiology procedures, such as central venous catheter placement [8, 22].

An abnormal, supraaortic variant of the typically subaortic course of the left BCV in the superior mediastinum was first described by Takada et al. [28]. Other left-BCV variants due to the vessel's abnormal embryogenesis have also been described: a double left $B C V$, formed when a doubled branch of the left $B C V$ courses inferoposteriorly to the ascending aorta before anastomosing with the right $\mathrm{BCV}$ or when the accessory vessel courses posteriorly to the trachea and oesophagus and merges with the azygos vein before draining into the SVC [24, 31]. In our study, we encountered the following left-BCV variants: subaortic left $B C V$, double left $B C V$, and another, previously unreported, variant where both vessels (the upper and lower one) coursed parallel to each other above the aortic arch [27]. Our records over a 5-year period showed left-BCV-only anomalies in $1.6 \%$ of the analysed CIED implantation procedures involving contrast venography.

During normal embryogenesis, the mature form of the right anterior cardinal vein forms the right SVC, whereas the left-sided vessel undergoes involution up to the $20^{\text {th }}$ week of gestation and, consequently, blood flow is redistributed to the right side. During abnormal embryogenesis the left anterior cardinal vein may not undergo involution, which leads to the presence of PLSVC. Over the last 16 years, during which CIED implantation procedures were conducted at our centre, this type of systemic vein anomaly was detected in $12(0.2 \%)$ cases, slightly less commonly than reported in other, comparable populations $[1,7,30]$.

Approximately $85 \%$ of adults diagnosed with PLSVC have two coexisting venous drainages. In our study, the PLSVC anomaly with two developed venous drainages (double SVC) was detected in $67 \%$ of cases. Out of these PLSVC cases, concomitant left-BCV agenesis with two independent SVC drainages due to a failure in the formation of the physiological bridge between the two anterior cardinal veins, was observed less commonly (50\%) than reported by other authors $[7,16,20,30]$.

A double SVC with the two vessels bridged by the left BCV was found in $4(33 \%)$ cases, out of all 12 detected cases of PLSVC. However, we believe that this type of double SVC may be more common due to the fact that some cases remain undetected; for instance, those cases where the continuation of the left subclavian vein into the left BCV favours smooth advancement of a cardiac lead into the right SVC, cases where cardiac leads are introduced via the right SVC, or cases that lack any diagnostic imaging that would visualise the presence of a double SVC [26].

In $10-20 \%$ of PLSVC cases, abnormal development of the right cardinal vein leads to right SVC agenesis. In our study, this type of venous drainage from both sides of the chest via a single PLSVC (naturally, via the left BCV) was observed in 4 (33\%) out of all cases of detected PLSVC [6, 23].

Having knowledge on the prevalence of left BCV anomalies along with the morphometric and topographic characteristics helps predict potential problems during transvenous procedures, such as central venous catheter and CIED, which employ systemic thoracic veins.

\section{Limitations of the study}

Our evaluation of the prevalence of presented left$-B C V$ variations was confined to a selected group of patients, namely those undergoing CIED implantation procedures with an accompanying venography. This may have caused the proportion of detected venous anomalies to be lower than their actual prevalence, both in populations undergoing CIED implantation and in the general population.

Failure to detect some of the developmental anomalies of mediastinal veins may be associated with the fact that venography of the left BCV is less common than that of its tributaries coursing through the left clavipectoral triangle. Moreover, during some procedures, the leads are advanced via the right $\mathrm{BCV}$.

\section{CONCLUSIONS}

The analysis of our study population of patients undergoing a CIED implantation procedure showed developmental anomalies of the left BCV alone to be rare, whereas left BCV agenesis in combination with PLSVC was more common. The asymptomatic 
character of left-BCV aberrations is usually the reason why they are detected only during certain transvenous procedures. Such aberrations in venous topography and morphometry may pose a challenge during CIED implantation procedures involving transvenous cardiac lead insertion.

\section{REFERENCES}

1. Biffi $M$, Bertini $M$, Ziacchi $M$, et al. Left superior vena cava persistence in patients undergoing pacemaker or cardioverter-defibrillator implantation: a 10-year experience. Chest. 2001; 120(1): 139-144, doi: 10.1378/ chest.120.1.139, indexed in Pubmed: 11451829.

2. Bowdle A. Vascular complications of central venous catheter placement: evidence-based methods for prevention and treatment. J Cardiothorac Vasc Anesth. 2014; 28(2): 358-368, doi: 10.1053/j.jvca.2013.02.027, indexed in Pubmed: 24008166.

3. Chen SJ, Liu KL, Chen HY, et al. Anomalous brachiocephalic vein: CT, embryology, and clinical implications. Am J Roentgenol. 2005; 184(4): 1235-1240, doi: 10.2214/ ajr.184.4.01841235, indexed in Pubmed: 15788602.

4. Curtil A, Tronc F, Champsaur G, et al. The left retro-aortic brachiocephalic vein: morphologic data and diagnostic ultrasound in 27 cases. Surg Radiol Anat. 1999; 21(4): 251-254, doi: 10.1007/BF01631395, indexed in Pubmed: 10549081.

5. Demos TC, Posniak HV, Pierce KL, et al. Venous anomalies of the thorax. Am J Roentgenol. 2004; 182(5): 1139-1150, doi: 10.2214/ajr.182.5.1821139, indexed in Pubmed: 15100109.

6. Gibelli G, Biasi S. Persistent left superior vena cava and absent right superior vena cava: not only an anatomic variant. J Cardiovasc Echogr. 2013; 23(1): 42-44, doi: 10.4103/22114122.117985, indexed in Pubmed: 28465883.

7. Goyal SK, Punnam SR, Verma G, et al. Persistent left superior vena cava: a case report and review of literature. Cardiovasc Ultrasound. 2008; 6: 50, doi: 10.1186/14767120-6-50, indexed in Pubmed: 18847480.

8. Granata A, Zanoli L, Trezzi M, et al. Anatomical variations of the left anonymous trunk are associated with central venous catheter dysfunction. J Nephrol. 2018; 31(4): 571-576, doi: 10.1007/s40620-017-0465-z, indexed in Pubmed: 29270845

9. Haq AA, Restrepo CS, Lamus D, et al. Thoracic venous injuries: an imaging and management overview. Emerg Radiol. 2016; 23(3): 291-301, doi: 10.1007/s10140-0161386-1, indexed in Pubmed: 26965007.

10. Bachleda JP. latrogenic injury to the superior vena cava and brachiocephalic vein. J Infect Dis Ther. 2014; 02(06), doi: 10.4172/2332-0877.1000169.

11. Kahkouee S, Sadr M, Pedarzadeh E, et al. Anomalous left brachiocephalic vein: important vascular anomaly concomitant with congenital anomalies and heart diseases. Folia Morphol. 2017; 76(1): 51-57, doi: 10.5603/ FM.a2016.0031, indexed in Pubmed: 27830886.

12. Ko SF, Huang $\mathrm{CC}, \mathrm{Ng} \mathrm{SH}$, et al. Imaging of the brachiocephalic vein. Am J Roentgenol. 2008; 191(3): 897-907, doi: 10.2214/AJR.07.3552, indexed in Pubmed: 18716126.

13. Ko SF, Ng SH, Fang FM, et al. Left brachiocephalic vein perforation: computed tomographic features and treatment considerations. Am J Emerg Med. 2007; 25(9): 1051-1056, doi: 10.1016/j.ajem.2007.06.013, indexed in Pubmed: 18022501.

14. Kobayashi M, Ichikawa T, Koizumi J, et al. Aberrant left brachiocephalic vein versus persistent left superior vena cava without bridging vein in adults: evaluation on computed tomography. Ann Vasc Dis. 2018; 11(4): 535-541, doi: 10.3400/avd.oa.18-00098, indexed in Pubmed: 30637011.
15. Kondrachuk O, Yalynska T, Tammo R. Double left brachiocephalic vein. Pediatr Cardiol. 2013; 34(3): 767-768, doi: 10.1007/s00246-012-0542-y, indexed in Pubmed: 23052674.

16. Kula S, Cevik A, Sanli C, et al. Persistent left superior vena cava: experience of a tertiary health-care center. Pediatr Int. 2011; 53(6): 1066-1069, doi: 10.1111/j.1442200X.2011.03443.x, indexed in Pubmed: 21810152.

17. Loukas M, Tobola MS, Tubbs RS, et al. The clinical anatomy of the internal thoracic veins. Folia Morphol. 2007; 66(1): 25-32, indexed in Pubmed: 17533591.

18. Nagashima M, Shikata F, Okamura T, et al. Anomalous subaortic left brachiocephalic vein in surgical cases and literature review. Clin Anat. 2010; 23(8): 950-955, doi: 10.1002/ca.21046, indexed in Pubmed: 20830788.

19. Povoski SP, Khabiri H. Persistent left superior vena cava: review of the literature, clinical implications, and relevance of alterations in thoracic central venous anatomy as pertaining to the general principles of central venous access device placement and venography in cancer patients. World J Surg Oncol. 2011; 9: 173, doi: 10.1186/14777819-9-173, indexed in Pubmed: 22204758.

20. Ratliff $\mathrm{HL}$, Yousufuddin $M$, Lieving WR, et al. Persistent left superior vena cava: case reports and clinical implications. Int J Cardiol. 2006; 113(2): 242-246, doi: 10.1016/j. ijcard.2005.08.067, indexed in Pubmed: 16318881.

21. Ruano CA, Marinho-da-Silva A, Donato P. Congenital thoracic venous anomalies in adults: morphologic MR imaging. Curr Probl Diagn Radiol. 2015; 44(4): 337-345, doi: 10.1067/j. cpradiol.2015.01.002, indexed in Pubmed: 25953438.

22. Salik E, Daftary A, Tal MG. Three-dimensional anatomy of the left central veins: implications for dialysis catheter placement. J Vasc Interv Radiol. 2007; 18(3): 361-364, doi: 10.1016/j.jvir.2006.12.721, indexed in Pubmed: 17377181.

23. Sheikh AS, Mazhar S. Persistent left superior vena cava with absent right superior vena cava: review of the literature and clinical implications. Echocardiography. 2014; 31(5): 674-679, doi: 10.1111/echo.12514, indexed in Pubmed: 24460570.

24. Shim MS, Kang MJ, Kim J, et al. Circumaortic left brachiocephalic vein: CT findings. J Korean Soc Radiol. 2010; 62(3): 207, doi: 10.3348/jksr.2010.62.3.207.

25. Standring S. Gray's anatomy. 39th ed. Hearts and great vessels. Chapter 60. Elsevier, Churchill, Livingstone, Edinburgh 2005: 1027.

26. Steckiewicz R, Kosior DA, Rosiak M, et al. The prevalence of superior vena cava anomalies as detected in cardiac implantable electronic device recipients at a tertiary cardiology centre over a 12-year period. HJC. 2016; 57(2): 101-106, doi: 10.1016/j.hjc.2016.03.003, indexed in Pubmed: 27445024.

27. Steckiewicz R, Świętoń EB, Stolarz P. A rare vascular anomaly in the form of double left brachiocephalic vein detected incidentally during cardiac implantable electronic device (CIED) placement. Folia Morphol. 2018; 77(1): 161-165, doi: 10.5603/FM.a2017.0065, indexed in Pubmed: 28703852.

28. Takada Y, Narimatsu A, Kohno A, et al. Anomalous left brachiocephalic vein: CT findings. J Comput Assist Tomogr. 1992; 16(6): 893-896, doi: 10.1097/00004728199211000-00012, indexed in Pubmed: 1430437.

29. Topcuoglu OM, Atceken Z, Ariyurek OM. Circumaortic doubled left brachiocephalic vein: a rare confusing variation. Surg Radiol Anat. 2015; 37(3): 315-318, doi: 10.1007/ s00276-014-1345-z, indexed in Pubmed: 25037739.

30. Webb WR, Gamsu G, Speckman JM, et al. Computed tomographic demonstration of mediastinal venous anomalies. Am J Roentgenol. 1982; 139(1): 157-161, doi: 10.2214/ ajr.139.1.157, indexed in Pubmed: 6979850.

31. Yigit $A E$, Haliloglu $M$, Karcaaltincaba $M$, et al. Retrotracheal aberrant left brachiocephalic vein: $\mathrm{CT}$ findings. Pediatr Radiol. 2008; 38(3): 322-324, doi: 10.1007/s00247-0070663-2, indexed in Pubmed: 17962930. 